\title{
Failed endovascular therapy for acute internal carotid artery occlusion from pituitary apoplexy: illustrative case
}

\author{
Marwah A. Elsehety, MD, ${ }^{1}$ Hussein A. Zeineddine, MD, ${ }^{2}$ Andrew D. Barreto, MD, MS, ${ }^{1}$ and Spiros L. Blackburn, $M D^{2}$ \\ ${ }^{1}$ Department of Neurology and ${ }^{2}$ Vivian L. Smith Department of Neurosurgery, University of Texas Medical School at Houston, Houston, Texas
}

BACKGROUND Large pituitary adenomas can rarely cause compression of the cavernous internal carotid artery (ICA) due to chronic tumor compression or invasion. Here, the authors present a case of pituitary apoplexy causing acute bilateral ICA occlusion with resultant stroke. Our middle-aged patient presented with sudden vision loss and experienced rapid deterioration requiring intubation. Computed tomography (CT) angiography revealed a large pituitary mass causing severe stenosis of the bilateral ICAs. CT perfusion revealed a significant perfusion delay in the anterior circulation. The patient was taken for cerebral angiography, and balloon angioplasty was attempted with no improvement in arterial flow. Resection of the tumor was then performed, with successful restoration of blood flow. Despite restoration of luminal patency, the patient experienced bilateral ICA infarcts.

OBSERVATIONS Pituitary apoplexy can present as an acute stroke due to flow-limiting carotid compression. Balloon angioplasty is ineffective for the treatment of this type of compression. Surgical removal of the tumor restores the flow and luminal caliber of the ICA.

LESSONS Pituitary apoplexy can be a rare presentation of acute stroke and should be managed with immediate surgical decompression rather than attempted angioplasty in order to restore blood flow and prevent the development of cerebral ischemia.

https://thejns.org/doi/abs/10.3171/CASE21370

KEYWORDS angiography; tumor; stenosis; stroke; intervention

Pituitary adenomas are benign, slow-growing tumors that most commonly present with headache, vision changes, or symptoms of endocrine dysfunction. Rarely, pituitary adenomas can invade the cavernous sinus and cause compression of the internal carotid artery (ICA). Here, we describe the first known case of pituitary apoplexy presenting as an acute stroke and being taken urgently to the angiography suite for intervention. Recognizing this presentation would expedite appropriate management, which involves surgical decompression via tumor resection rather than endovascular interventions.

\section{Illustrative Case}

A 60-year-old female presented with 2 hours of sudden-onset vision loss and vomiting. She had last been seen in normal condition 5 hours prior. She was initially awake and responding appropriately but progressively became more lethargic, and a noncontrast computed tomography (CT) scan of her head was obtained, which revealed a large, nonhemorrhagic pituitary mass. She was transferred to our facility for neurosurgical evaluation and upon arrival was noted to be unresponsive with a Glasgow Coma Scale score of 5 . She was immediately intubated for airway protection, and a code stroke was activated.

An urgent CT scan of the patient's head revealed a large sellar and suprasellar mass concerning for pituitary macroadenoma, measuring $3.5 \mathrm{~cm}$ by $3.1 \mathrm{~cm}$ by $3.7 \mathrm{~cm}$ (Fig. 1). CT angiography (CTA) of the head and neck revealed severe flow limitation starting at the cavernous ICAs bilaterally (Fig. 2). Due to artifact from the anterior clinoids, it was unclear whether there was a carotid thrombus or carotid stenosis secondary to tumor mass effect. CT perfusion revealed a significant perfusion delay in the anterior circulation with a mismatch volume of $474 \mathrm{~mL}$ in the bilateral anterior cerebral artery (ACA) and middle cerebral artery (MCA) territories (Fig. 3). The patient was taken for emergent cerebral angiography. A cerebral angiogram revealed severe flow-limiting stenosis of the bilateral

ABBREVIATIONS ACA = anterior cerebral artery; $C T=$ computed tomography; $C T A=$ computed tomography angiography; $I C A=$ internal carotid artery; $M C A=$ middle cerebral artery.

INCLUDE WHEN CITING Published September 6, 2021; DOI: 10.3171/CASE21370.

SUBMITTED June 21, 2021. ACCEPTED July 22, 2021.

(c) 2021 The authors, CC BY-NC-ND 4.0 (http://creativecommons.org/licenses/by-nc-nd/4.0/). 


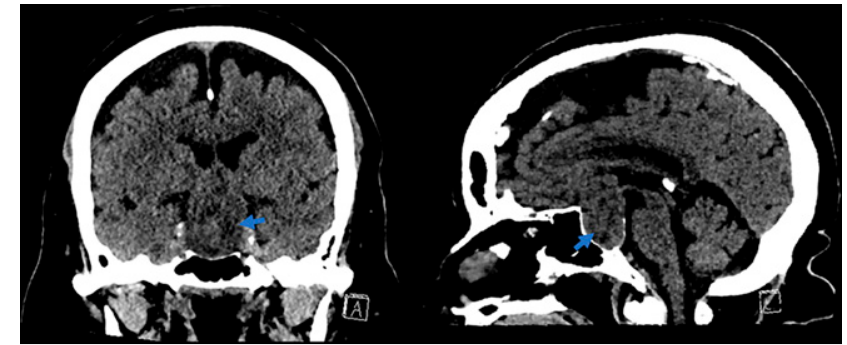

FIG. 1. CT of the head showing a large, $3.5-\mathrm{cm} \times 3.1-\mathrm{cm} \times 3.7-\mathrm{cm}$ sellar and suprasellar mass. Blue arrows highlight the expanded sella by the tumor.

ICAs at the level of the anterior clinoids (Fig. 4A and B). No intraluminal thrombus was visualized.

In the interventional suite, balloon angioplasty of the right supraclinoid ICA was performed without sustained improvement of luminal diameter and with persistent flow limitation. The patient was then taken for an emergent transsphenoidal hypophysectomy. Intraoperatively, the sellar contents decompressed rapidly, and the tumor tissue was noted to be liquefied and necrotic, consistent with an infarcted rather than a hemorrhagic mass. After hypophysectomy, the patient was taken directly for angiography, and a repeat cerebral angiogram revealed resolution in the clinoid ICA stenoses bilaterally with resolution of intracranial flow limitation and normal circulation times (Fig. $4 \mathrm{C}$ and D).

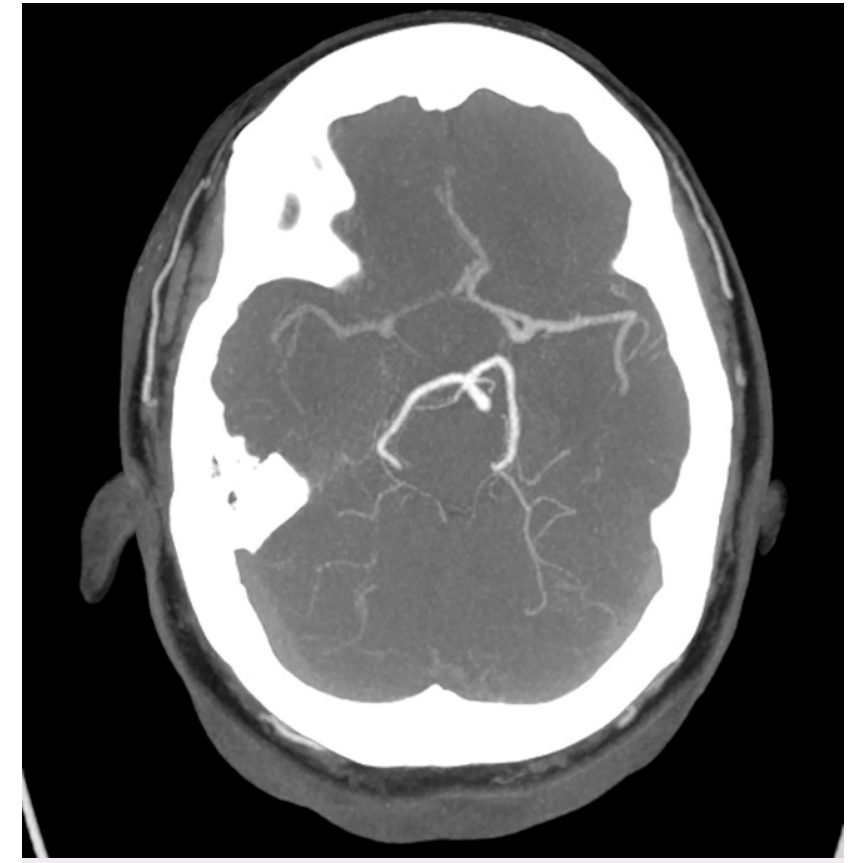

FIG. 2. CTA showing poor opacification of the intracranial anterior circulation, as compared with the posterior circulation.
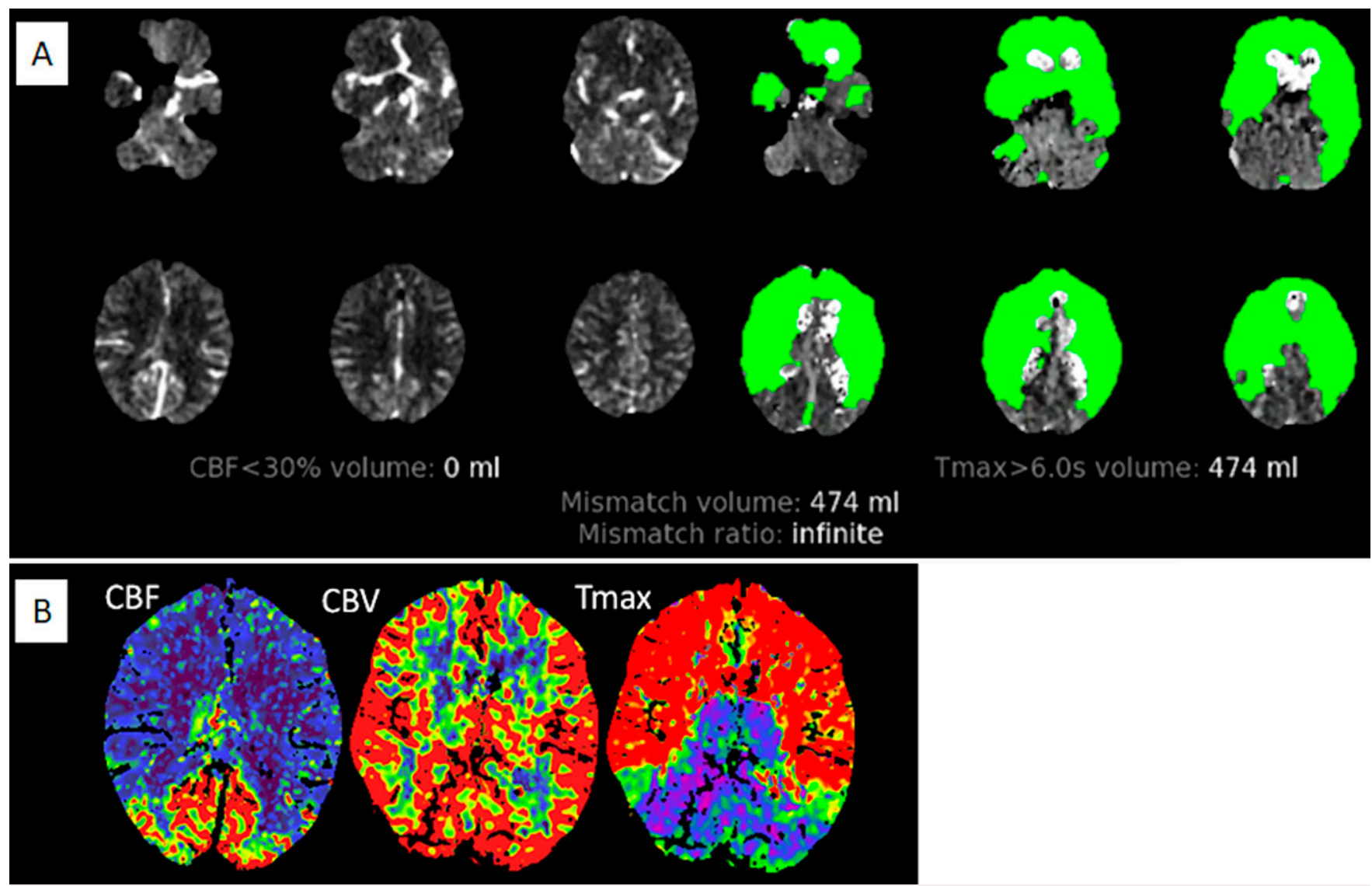

FIG. 3. Rapid CT perfusion (A) showing a large mismatch volume of $474 \mathrm{~mL}$ in the bilateral ICA territories and CT perfusion maps (B) showing delayed anterior cerebral blood flow (CBF), cerebral blood volume (CBV), and time to maximum (Tmax). 


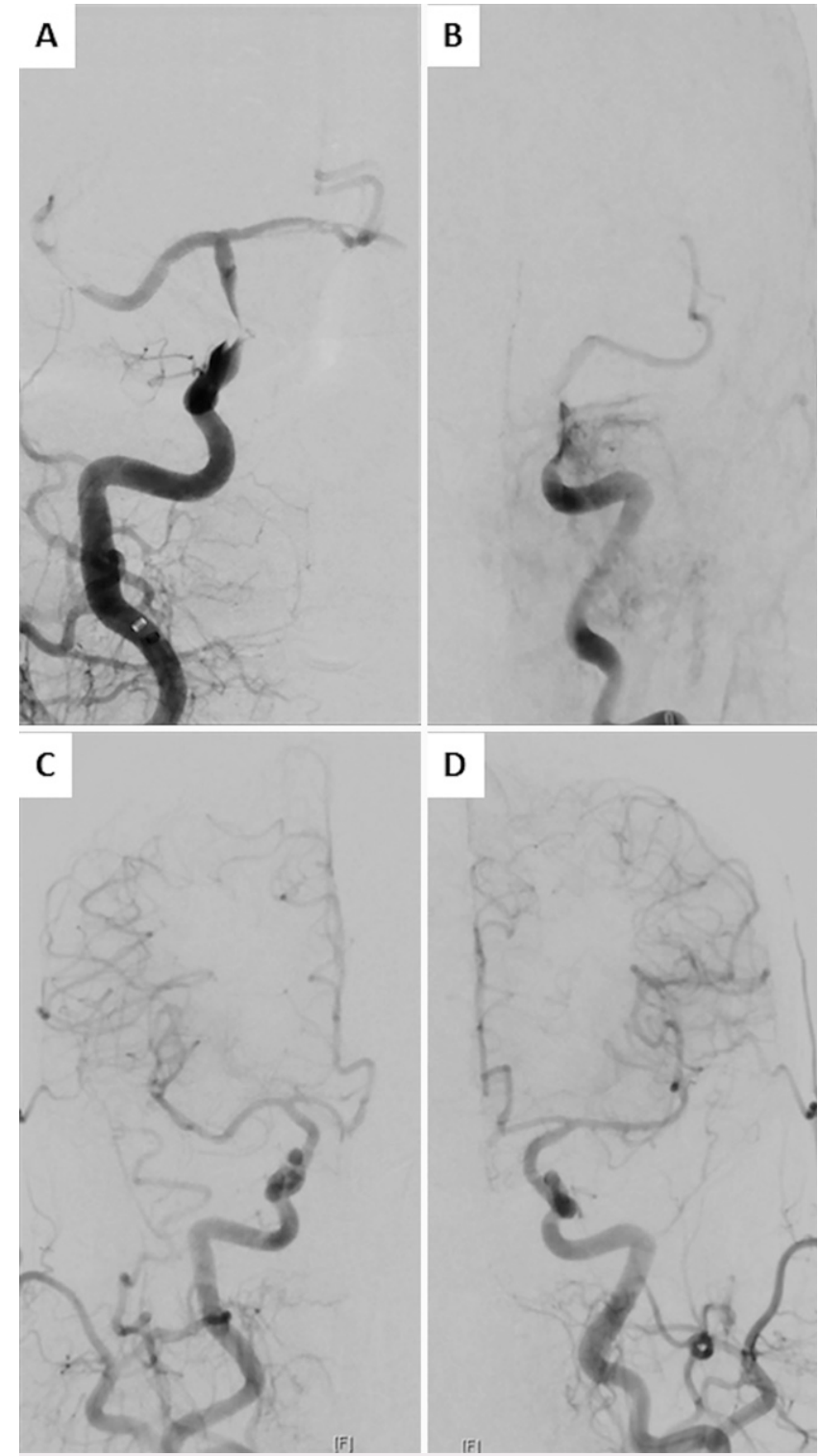

FIG. 4. Anteroposterior (A) right and (B) left common carotid artery angiograms showing stenosis of the clinoid ICAs and extremely delayed filling of the bilateral ACA and MCA. After transsphenoidal resection, the patient was returned to the angiography suite, and a postoperative angiogram showed normalization of flow through the (C) right and (D) left ICAs.

The histopathology report confirmed a clinically nonfunctioning pituitary adenoma. Postoperatively, the patient remained comatose. Magnetic resonance imaging of the brain at 24 hours revealed extensive anoxic injury of the bilateral anterior cerebral hemispheres and subcortical structures, with relative sparing of the posterior cerebral hemispheres, brainstem, and cerebellum (Fig. 5). The devastating injury was discussed with the family, and they opted to pursue palliative care and comfort measures. The patient died shortly after extubation.

\section{Discussion}

\section{Observations}

Our case illustrates an acute bilateral ICA stroke secondary to pituitary tumor apoplexy. This case should alert physicians to a rare cause of acute stroke. Endovascular therapy was initially attempted with angioplasty after it was noted that there was no thrombus. However, this did not deliver sustained carotid patency. An intracranial stent was considered, but without a clear understanding of the compressive force from the infarcted tumor, this was not attempted. Surgical decompression was ultimately successful at restoring carotid flow bilaterally.

Pituitary adenomas can frequently encase the ICA, but they rarely cause arterial compression, especially when compared with other sellar and parasellar masses that invade the cavernous sinus. ${ }^{1}$ Symptomatic carotid artery stenosis secondary to compression by a pituitary adenoma is most commonly the result of pituitary apoplexy, with a number of reports of this previously described in the literature. ${ }^{2-6}$ The majority of these have resulted in unilateral ICA compression, with only a handful of the cases resulting in bilateral carotid artery compression. ${ }^{2}$ There have been six reported cases of symptomatic ICA compression by nonapoplectic pituitary adenomas, with only one other reported case resulting in bilateral carotid compression. ${ }^{7,8}$ Clinical presentations have ranged from transient ischemic attacks to altered consciousness and rapid clinical deterioration as in our patient, and the outcomes have ranged from complete resolution of symptoms to death.

\section{Lessons}

Pituitary apoplexy can be a rare presentation of acute stroke and should be managed with immediate surgical decompression rather than attempted angioplasty in order to restore blood flow. Endovascular treatment delayed surgical decompression and likely contributed to increased cerebral ischemia. Although acute vascular compression secondary to a pituitary macroadenoma is rare, it is important to consider prompt surgical decompression to prevent the development of cerebral ischemia and improve the patient's outcome.

\section{References}

1. Molitch ME, Cowen L, Stadiem R, Uihlein A, Naidich M, Russell E. Tumors invading the cavernous sinus that cause internal carotid artery compression are rarely pituitary adenomas. Pituitary. 2012; 15(4):598-600.

2. Banerjee C, Snelling B, Hanft S, Komotar RJ. Bilateral cerebral infarction in the setting of pituitary apoplexy: a case presentation and literature review. Pituitary. 2015;18(3):352-358.

3. Rosenbaum TJ, Houser OW, Laws ER. Pituitary apoplexy producing internal carotid artery occlusion. Case report. J Neurosurg. 1977; 47(4):599-604.

4. Dogan S, Kocaeli H, Abas F, Korfali E. Pituitary apoplexy as a cause of internal carotid artery occlusion. J Clin Neurosci. 2008;15(4): 480-483.

5. Abbas MS, AlBerawi MN, Al Bozom I, Shaikh NF, Salem KY. Unusual complication of pituitary macroadenoma: a case report and review. Am J Case Rep. 2016;17:707-711.

6. Ahmed SK, Semple PL. Cerebral ischaemia in pituitary apoplexy. Acta Neurochir (Wien). 2008;150(11):1193-1196.

7. Rezaei O, Sharifi G, Ebrahimzadeh K, Hallajnejad M. Symptomatic occlusion of internal carotid artery caused by pituitary macro adenoma among the patients without apoplectic signs; case report and review of the literature. Int J Cancer Manag. 2016;10(3):e5301.

8. Rey-Dios R, Payner TD, Cohen-Gadol AA. Pituitary macroadenoma causing symptomatic internal carotid artery compression: surgical treatment through transsphenoidal tumor resection. J Clin Neurosci. 2014;21(4):541-546. 


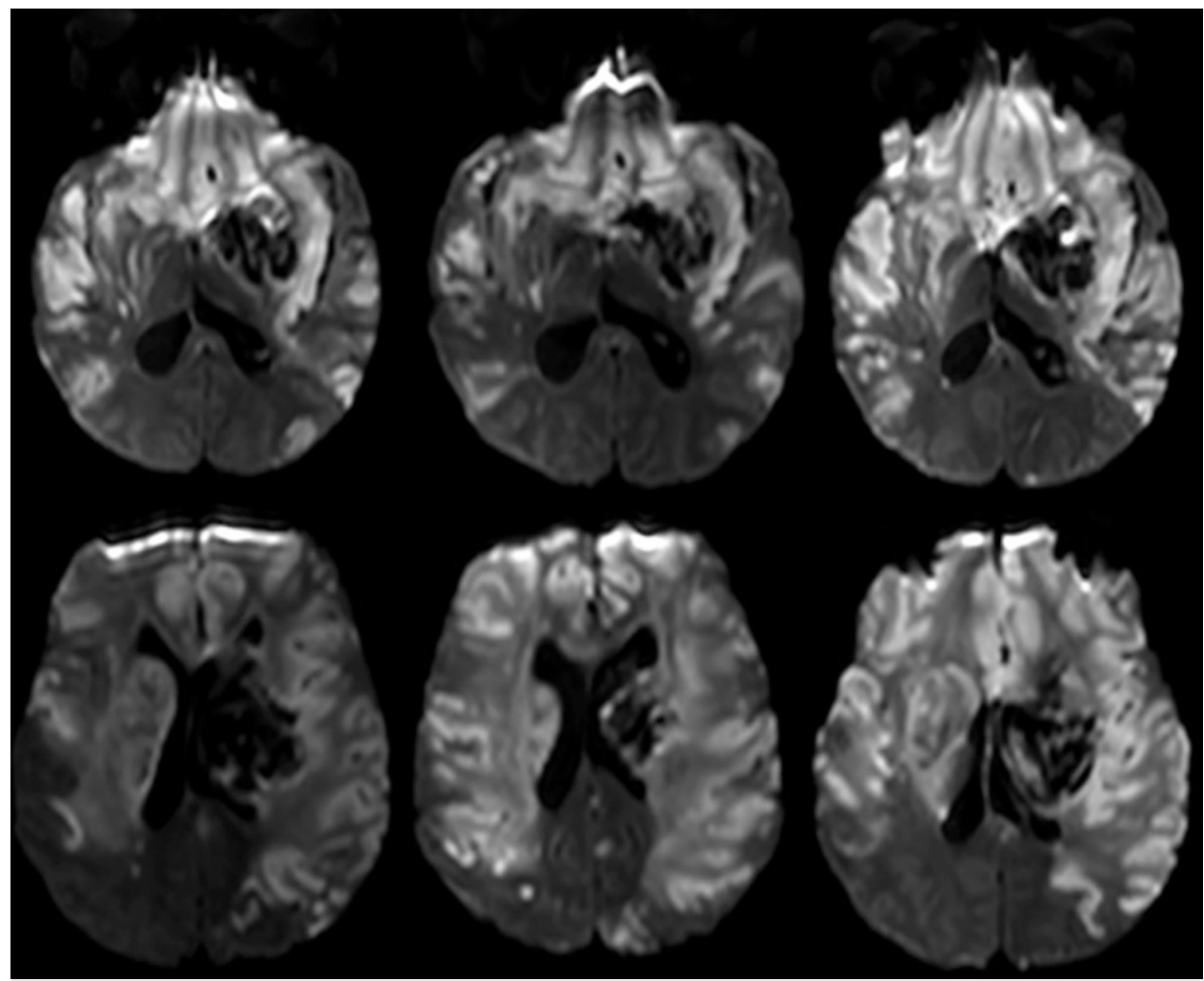

FIG. 5. Postoperative magnetic resonance imaging showing anoxic injury of the bilateral ICA territories, with relative sparing of the territories supplied by the posterior circulation.

\section{Disclosures}

The authors report no conflict of interest concerning the materials or methods used in this study or the findings specified in this paper.

\section{Author Contributions}

Conception and design: all authors. Acquisition of data: Blackburn, Zeineddine, Barreto. Analysis and interpretation of data: Blackburn, Zeineddine. Drafting the article: Elsehety, Zeineddine. Critically revising the article: all authors. Reviewed submitted version of manuscript: all authors. Approved the final version of the manuscript on behalf of all authors: Blackburn. Study supervision: Blackburn, Barreto.

\section{Correspondence}

Spiros L. Blackburn: University of Texas Medical School at Houston, Houston, TX. spiros.blackburn@uth.tmc.edu. 